\title{
One Hour Ahead Load forecasting Using Support Vector Machines
}

\author{
Tahir Nadeem ${ }^{[1]}$, M.Arif ${ }^{[2]}$, Haider Ali ${ }^{[3]}$, S.Wajahat Ali Shah ${ }^{[4]}$, Usman Khalid $^{[5]}$ \\ ${ }^{1}$ (UET,Taxila,Pakistan) \\ 2,3, 4, 5 (CIIT Abbottabad,Pakistan)
}

\begin{abstract}
In this paper a Novel Neural Network Technique is presented using Support Vector Machine to develop a Short Term Load Forecasting (STLF) model for coming 24 hour load prediction considering various weather factors (Temperature \& Humidity). In the STLF, future Load is planned up to weak ahead. Many approaches are used to develop a short term load forecasting model consisting of traditional, Artificial Intelligence (AI) and hybrid model. However there is intense need to develop a more accurate and efficient model for STLF. Detailed experiments were performed on AEMO and California Electricity market data. The results of the proposed technique have been compared with ANN models. Experimental results show that proposed technique is better in terms of accuracy, prediction \& training time.
\end{abstract}

Keywords: Artificial Intelligence, Neural Network, Regression, STLF, SVM

\section{Introduction}

Short term load forecasting is first and vital step in power system operation and control [1]. It has too much significance in power system planning and market based power system. Accurately forecasting load helps the utility company to make its operation and unit commitment economical $[2,3]$. Good prediction of electric load resolves the issues regarding to the reliability, security and efficiency of the power system [4]. Accuracy and time is more important parameters in the load forecasting. Under prediction of short term load forecasting leads to insufficient reserve capacity preparation and hence increase the operation cost by using the expensive peaking units [5]. Over prediction of STLF involves in allocating the large reserve capacity which is also related to high operation cost. Many techniques and approaches has been used to solve the short term load forecasting problems consisting of traditional auto regression , method of least square, artificial intelligence like neural network ,fuzzy logic ,support vector machine , Particle Swarm Optimization [6-13] and many others which are combination of two or more traditional, artificial intelligence or both techniques .STLF problems are still challenges for the researcher traditional techniques are used so of that they solution of the algebraic equation become difficult as the model complexity increases. Now a day's Artificial Intelligence based techniques are more frequently used for the STLF problems [14]. Artificial Neural Network based STLF models are most promising tool used by the researcher but as there are numerous hidden layers in the neural network models making it slow in the training and testing phase. There are numerous ANN flavours used for the STLF. Another novel neural technique which attains a great attention of the researcher is Support Vector Machine which is more fast and accurate than ANN [15].

In this research the Support Vector Machine is applied for the Next hour Load prediction considering weather factors. Models for Australian Electricity Market operator data and California state electricity market Data are developed. Two STLF models are developed first is next hour load prediction and second is next 24 hour load prediction. Temperature and humidity are used as inputs of the models. The developed model consists of previous 24 hour data along with the maximum temperature of the forecasted day.

\section{Support Vector Machine}

Support Vector Machine is a supervised learning tool in which the training error is assumed fixed and training the model with pre-defined data. SVM tool is used for both classification and regression problems and it is based on statistical learning theory .In SVM some sample data as an input is given and its output function is used to predict some feature of the future data.

In many conventional and mathematical techniques the models are known but there are many real world applications for which the provided information is not enough to define mathematical model, more data points for the modelling are required. Conventional techniques use large data set in the training, error is less while for small data size error is maximum.

In learning techniques important factor is to develop a model for sparse data. (Vapnik, 1995) developed the Support Vector Machine to solve the classification and regression problems and it is based on the statistical learning theory. Support Vector Machine is novel neural network technique using the structural risk minimization framework for its learning unlike conventional techniques that use the Norm-1 and Norm-2. 
Support vector machine algorithm widely used in many engineering applications with two major applications SVM in classification problems and Regression problems.

Support Vector Regression for the STLF models is used for the proposed system. A data set $D=\left\{\left(a_{1}, y_{1}\right),\left(a_{2}, y_{2}\right) \ldots \ldots \ldots \ldots \ldots\left(a_{n}, y_{n}\right)\right\}$ consists of M pairs of data in which "a's" are input vectors and " $y$ 's" are corresponding outputs so optimization function is:

$f(a, v)=\sum_{i=1}^{n} V_{i} \phi(a)$

Where $\phi(a)$ is called feature in nonlinear classification and also including the bias term ' $\mathrm{b}$ '. The function $f(x, w)$ is explicitly written as function $\mathrm{w}$.

$f(a, v)=\sum_{i=1}^{n} V^{T} A+b$

In regression typical some error of approximation is used instead of hyper plane as in SVM classification problem.

- $\quad$ Norm $1|Y-f|$

- $\quad$ Norm $2|Y-f|^{2}$

Vapnik general type loss function e-infectivity zone is in equation (3) .

$$
\begin{array}{r}
|Y-f(a, v)|=\{0 \text { if } \mid Y-f(a, v) \leq e \\
|Y-F(a, v)|-e \text { otherwise }
\end{array}
$$

The loss is zero if the difference between predicted $f(a, v)$ and measure value ' $\mathrm{Y}$ ' is zero or less than e .Vapnik introduce ' $\mathrm{e}$ ' tube of predicted value is within the tube loss is zero .For all other predicted points loss is equal to zero.

In order to perform SVM regression new empirical risk is

$$
\text { Remp }=1 / M \sum_{i=0}^{M}\left(y i-v^{T} A_{i}-b\right)
$$

In SVM regression the objective is to minimize the loss Remp.Estimating a linear hyper plan

$$
\begin{aligned}
& f(a, v)=v^{T} a+b \\
& R=1 / 2\|v\|^{2}+C \sum_{i=1}^{M}\left|Y_{i}-f\left(a_{i}, w\right)\right|
\end{aligned}
$$

It is ridge expression example.

$|Y-f(v, w)|-e=\varepsilon+$ above tube

$|Y-f(v, w)|-e=\varepsilon-$ below tube

Then the objective function is

$$
R\left(v, \varepsilon+, \varepsilon^{-}\right)=\left[1 / 2\|\nu\|^{2}+C \sum_{i=0}^{M} \varepsilon^{+}+C \sum_{i=0}^{M} \varepsilon^{-}\right.
$$

Subject to: 
$Y_{i}-v^{T} A_{i}-b \leq \varepsilon+e$

$v^{T} A_{i}+b-y_{i} \leq \varepsilon+e$

$\varepsilon>0$

Epsilons are slack variables for above and below Epsilon tube. Both are positive than Lagrange multipliers $\alpha i$ and $\beta i \&$ epsilons will be non-zero points for training data. For data inside tube both multipliers are equal to zero.C is trade-off between Epsilon and weight vector .An increase in C leads the decrease in approximation error. This can be achieved only by weight vectors however increase in C does not give a good approximation model. A constrained optimization problem is constituted as

$O_{p}\left(v, b, \varepsilon+, \varepsilon^{-}, \alpha_{i}, \alpha^{*}, \beta_{i}, \beta_{i}^{*}\right)=1 / 2^{v^{T}} v+C \sum_{i=1}^{M} \varepsilon^{+} \sum_{i=1}^{M} \varepsilon^{-}$

-......- $\sum_{i=1}^{M} \alpha i\left[y_{i}-v^{T} a_{i}-b+\varepsilon^{+} \varepsilon^{*}\right]-\sum_{i=1}^{M} \alpha i\left[v^{T} a_{i}+b-y_{i}+\varepsilon^{+} \varepsilon^{*}\right]-\sum_{i=1}^{M} \beta_{i} \varepsilon^{*}+\beta_{i} \varepsilon_{i}$

A primal variable lagrangian $O_{p}\left(v, b, \varepsilon+, \varepsilon-, \alpha_{i}, \alpha^{*}, \beta_{i}, \beta_{i}^{*}\right)$ is minimized with respect to primal variables $\mathrm{v}$,b epsilon $\varepsilon$ and $\varepsilon^{*}$.

Calculating the lagrange multipliers, $\alpha_{i}, \alpha^{*}$ gives an optimal desired weight vector for regression hyper plan

$V_{0}=\sum_{i=1}^{M}\left(\alpha_{i}^{*}-\alpha_{i}\right) a_{i}$

And the optimal bias term for regression hyper plan.

$b_{0}=1 / 2 \sum_{i=1}^{M}\left(y_{i}-v_{0}{ }^{T} a_{i}\right)$

The best regression hyper plan is obtained

$Z=f(a, w)=v^{T} a+b$

\section{Stlf Execution Flow}

The following steps describe the execution of proposed technique.

1. Kernel function selection

2. Selecting the shape of the kernel linear, RBF or Gaussian kernel.

3. Choosing the penalty factor $\mathrm{C}$ and selecting the desired accuracy by setting $\mathbf{e}$.

4. Solving the Optimization function

\section{Results}

STLF model for the Australian Energy Market operator utility company using Support Vector Machine electricity load values, temperature and humidity. A set of training data for the year 2000 and 2001 is used for testing and training of the algorithm. Daily maximum temperature and humidity is included in the model as input parameter. "Fig. 1" shows the next 24 hour result of the model the difference between actual and predicted and the Absolute percentage error.

\begin{tabular}{|c|c|c|c|c|}
\hline NO & Actual Load (MW) & Predicted(MW) & $\begin{array}{c}\text { Difference } \\
(\mathbf{M W})\end{array}$ & APE\% \\
\hline 1 & 6735.8 & 6818.7 & -82.9 & 1.23 \\
\hline 2 & 8484.2 & 8186.1 & 298.1 & 3.51 \\
\hline 3 & 8383.8 & 8405.4 & -21.6 & 0.25 \\
\hline 4 & 8351.8 & 8485.3 & -133.5 & 1.59 \\
\hline 5 & 8471.5 & 8365.5 & 106 & 1.25 \\
\hline 6 & 7646.4 & 7311.6 & 334.8 & 4.37 \\
\hline
\end{tabular}




\begin{tabular}{|c|c|c|c|c|}
\hline 7 & 7532.8 & 7089.7 & 443.1 & 5.88 \\
\hline 8 & 7317.7 & 7139.4 & 178.3 & 2.43 \\
\hline 9 & 7084.3 & 6905.2 & 179.1 & 2.52 \\
\hline 10 & 8467.9 & 8267 & 200.9 & 2.37 \\
\hline 11 & 8493.7 & 8470.6 & -140.3 & 0.27 \\
\hline 12 & 8436.6 & 8576.9 & 82.6 & 0.97 \\
\hline 13 & 8466.9 & 8384.3 & -60.8 & 0.71 \\
\hline 14 & 8458.2 & 8519 & -114.1 & 1.34 \\
\hline 15 & 8457.9 & 8572 & -14.9 & 0.17 \\
\hline 16 & 8476.2 & 8491.1 & 578.1 & 7.24 \\
\hline 17 & 7978.2 & 7400.1 & -227.1 & 2.69 \\
\hline 18 & 8416 & 8643.1 & -924.8 & 13.01 \\
\hline 19 & 7085.5 & 8010.3 & -60.8 & 0.89 \\
\hline 20 & 6804 & 6864.8 & -18.5 & 0.28 \\
\hline 21 & 6423.3 & 6441.8 & -100.7 & 1.63 \\
\hline 22 & 6147.7 & 6248.4 & -58.4 & 0.99 \\
\hline 23 & 5873.5 & 5931.9 & -93.1 & 1.62 \\
\hline 24 & 5723.7 & 5816.8 & & \\
\hline
\end{tabular}

Figure I: Actual and Predicted data comparision

The result of next 24 hour load is shown graphically in "fig.2".

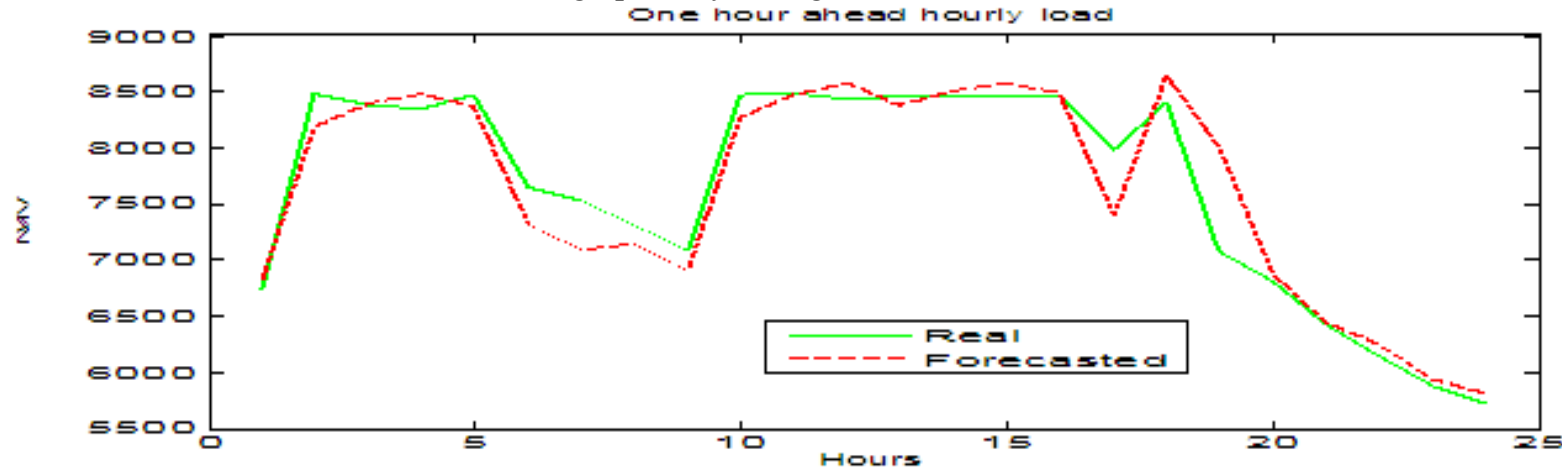

Fig 2. Real and Forecasted 24 hour Load

\section{Conclusions}

The Next hour electric load forecasting plays a vital role in the power system operation, commitment of generating units and energy management. Results show that the proposed STLF has better response in terms of accuracy, error \& training time. The results are accurate with less percentage of error(less than 2 precent average error). The system requires short time for training and provides accurate results.

\section{References}

[1] Miloš Božić, Miloš Stojanović, “Application of SVM Methods for Mid-Term Load Forecasting” SERBIAN JOURNAL OF ELECTRICAL ENGINEERING Vol. 8, No. 1, February 2011, 73-83

[2] N.M. Pindoriya, S.N. Singh, S.K. Singh "One-Step-Ahead Hourly Load ForecastingUsing Artificial Neural Network" Third international conference on power system kharaghpur india December 27-29,2009 Page No 177

[3] M. O. Oliveira, Marzec, Bordin, A.S. Bretas, D. Bernardon,. “Climate Change Effect on Very Short-TermElectric Load Forecasting” Paper accepted for presentation at the 2011 IEEE Trondheim PowerTech.

[4] A.Azadeh, S.F.Ghadrei, B.Pourvalikhan Nokhandan" One day ahead load forecasting for electricity market of Iran by ANN" IEEE POWERENG 2009.

[5] Shu Fan, Senior Member, IEEE, and Rob J Hyndman "Short-term Load Forecasting Using Semi-parametric Additive Models" IEEE POWER \& ENERGY SOCIETY GENERAL MEETING 2011

[6] C. Bhurtun, I. Jahmeerbacus, and C. Jeewooth "SHORT TERM LOAD FORECASTING IN MAURITIUS USING NEURAL NETWORK" Proceedings of the 8th Conference on the Industrial and Commercial Use of Energy.2011.

[7] M. F. I. Khamis, Z. Baharudin, N. H. Hamid, M. F. Abdullah, S. Solahuddin "Electricity Forecasting For Small Scale Power System Using Artificial Neural Network "The 5th International Power Engineering and Optimization Conference (PEOCO2011), Shah Alam, Selangor, Malaysia : 6-7 June2011.

[8] Gwo-Ching Liao IEEE Member "An Improved Fuzzy Neural Networks Approach for Short-Term Electrical Load Forecasting" Proceedings of 8th Asian Control Conference (ASCC)Kaohsiung, Taiwan, May 15-18, 2011.

[9] Rui Ma 1, Fei Jiang 1, Junying Song 2, Huihua Chen 2, Hanbing Dong3 "The Short-term Load Forecasting Based on the Rate of Load Fluctuation" Fourth International Conference on Intelligent Computation Technology and Automation in 2011.

[10] Aneesh Nair, S.K.Joshi "Short Term Load Forecasting Using Probabilistic Neural Network Based Algorithm" International Conference on Computational Intelligence and Communication Networks 2010.

[11] Yuan Ningl, Yufeng Liu I, Huiying Zhang I, Qiang Ji 2 "Comparison of different BP neural network models for short-term load forecasting'IEEE 2010. 
[12] W.Yuill,R.Kgokong,S.Chowdhury, S.P.Chowdhury "Application of Adaptive Neuro Fuzzy Inference System (ANFIS) based Short Term Load Forecasting in South African Power Networks" UPEC2010 31st Aug - 3rd Sept 2010.

[13] Arash Ghanbari Esmaeil Hadavandi Salman Abbasian-Naghneh "Comparison of Artificial Intelligence based Techniques for Short Term Load Forecasting". Third International Conference on Business Intelligence and Financial Engineering 2010.

[14] Wen-Chen Chu, Member, IEEE, Yi-Ping Chen, Member, IEEE, Zheng-Wei Xu, and Wei-Jen Lee, Fellow, IEEE "Multiregion Short-Term Load Forecasting in Consideration of HI and Load/Weather Diversity" IEEE TRANSACTIONS ON INDUSTRY APPLICATIONS, VOL. 47, NO. 1, JANUARY/FEBRUARY 2011

[15] Hemen Showkati, Amir H. Hejazi, Sajad Elyasi “Short Term Load Forecasting Using Echo State Networks” IEEE 2010. 